\title{
Resultados del diagnóstico temprano de sordera en el recién nacido con factores de riesgo en el Centro Médico ABC
}

\author{
Results of early diagnosis of deafness in newborns \\ with risk factors at the $A B C$ Medical Center
}

\author{
Rossela de Jesús Vega Castro, ${ }^{\star}$ Cecilia Alejandra Carranza Alva, ${ }^{\ddagger}$ René Toral Martiñón, $\$$ \\ Paul Shkurovich Bialik," Marivis Helena Polania Rodríguez," Roberto Richheimer Watson"
}

\section{RESUMEN}

Introducción: La prevalencia de discapacidad auditiva en los recién nacidos prematuros o con bajo peso al nacer es de 1-2\%, diez veces más alta que la encontrada en los recién nacidos de término. Objetivo: Conocer el resultado del diagnóstico temprano de sordera en el recién nacido con factores de riesgo en el Centro Médico ABC. Métodos: Estudio observacional, descriptivo, retrospectivo, retrolectivo y transversal. Los datos audiológicos, farmacológicos y clínicos fueron extraídos del expediente electrónico de pacientes hospitalizados en la Unidad de Terapia Intensiva y de Cuidados Intermedios Neonatales (UTIN-UCIN) del Centro Médico ABC Campus Santa Fe, entre enero de 2010 y mayo de 2018, y a quienes se les realizó tamiz auditivo neonatal mediante Emisiones Otoacústicas (EOA). El análisis estadístico se calculó mediante frecuencias absolutas y relativas, y se utilizó el paquete estadístico SPSS v21.0. Resultados: De 11,698 recién nacidos vivos, 1,735 pacientes ingresaron a la UCIN-UTIN, de los cuales 1,422 cumplieron con los criterios de selección; de ellos el $2.5 \%$ no pasó la primera prueba, $0.6 \%$ no pasó la segunda prueba y $0.07 \%$ presentó alteración en estudios subsecuentes. Conclusiones: Es de suma importancia la detección oportuna de sordera en el recién

\begin{abstract}
Introduction: The prevalence of hearing impairment in preterm or low weight infants is about 1-2\%, 10 times higher than found in full-term patients. Objective: To identify early diagnosis results of deafness in newborns with risk factors at the ABC Medical Center. Methods: Observational, descriptive, retrospective, retrolective and transversal study. Audiological, pharmacological and clinical data were extracted from electronic files of hospitalized patients in Neonatal Intensive Care Unit (NICU) and Neonatal Intermediate Care Unit in The ABC Medical Center from January 2010 to May 2018 with neonatal hearing screening performed by Otoacoustic Emissions (OAE). The statistical analysis was calculated using absolute and relative frequencies, the statistical package used was SPSS v21.0. Results: From 11,698 live births, 1,735 patients were admitted to NICU, 1,422 met the selection criteria, $2.5 \%$ of them failed to pass the first test, $0.6 \%$ failed the second test and $0.07 \%$ presented auditive impairment in subsequent studies. Conclusions: Early detection of deafness is very important, especially in newborns with multiple risk factors for auditory impairment. At the $A B C$ Medical Center, the neonatal auditory screening is performed routinely,
\end{abstract}

\footnotetext{
* Neurofisiólogo clínico.

‡ Médico adscrito al Servicio de Neurofisiología y Miembro del Centro Neurológico.

$\S$ Médico adscrito al Servicio de Audiología, Neurofisiología.

" Jefe del Servicio de Neurofisiología.

" Médico Pediatra, Neonatólogo.

\section{Centro Médico ABC.}

\section{Recibido para publicación: 31/05/2019. Aceptado: 15/11/2019.}

\section{Correspondencia: René Toral Martiñón}

Centro Médico ABC. Sur 136 Núm. 116, Col. Las Américas, 01120,

Álvaro Obregón, Ciudad de México, México. Tel-Fax: (55) 5273-2072

E-mail: rtoralm@gmail.com

\author{
Abreviaturas: \\ $\mathrm{HL}=$ Hearing level (por sus siglas en inglés). \\ $E O A=$ Emisiones otoacústicas. \\ PEATC $=$ Potenciales evocados auditivos de tallo cerebral. \\ UTIN = Unidad de Terapia Intensiva Neonatal. \\ UCIN = Unidad de Cuidados Intermedios Neonatales. \\ OMS = Organización Mundial de la Salud. \\ SDG = Semanas de gestación .
}

Este artículo puede ser consultado en versión completa en: www.medigraphic.com/analesmedicos 
nacido, sobre todo en aquéllos que presentan factores de riesgo de compromiso auditivo. En el Centro Médico ABC se realiza de manera rutinaria el tamiz auditivo neonatal y, en conjunto con el Servicio de Neonatología, se ha logrado la intervención oportuna para disminuir en gran medida el efecto de los factores de riesgo en el resultado auditivo de los pacientes.

Palabras clave: Tamiz auditivo, sordera, hipoacusia, recién nacidos, factores de riesgo.

Nivel de evidencia: III

\section{INTRODUCCIÓN}

La Organización Mundial de la Salud (OMS) define a la hipoacusia como una deficiencia auditiva que describe la pérdida de la audición en uno o ambos oídos, mientras que la sordera corresponde a la pérdida completa de la habilidad de oír en uno o ambos oídos. ${ }^{1}$

La pérdida de la audición congénita es frecuente al nacer, sobre todo en pacientes del grupo pediátrico con factores de riesgo perinatales importantes. El compromiso de la audición durante la etapa neonatal o infancia temprana condiciona las alteraciones en la adquisición y desarrollo del lenguaje, la interacción intelectual y el compromiso del aprendizaje, así como la adquisición de seguridad y participación social. ${ }^{2,3}$

La American National Standards Institute y la clasificación clásica de Davis y Silverman reportan la deficiencia auditiva y la clasifican según el tipo que pueda ser (conductiva o neurosensorial) y el grado (superficial, media, profunda y anacusia audiométrica). Un niño presenta problema de audición cuando el umbral para ambos oídos es igual o superior a 15 dB HL y en un adulto es de 25 dB HL. ${ }^{1}$

El umbral auditivo es la menor cantidad de energía perceptible en la zona de lenguaje, ésta se encuentra entre los 300 y $3,000 \mathrm{~Hz}$. Se reporta una audición normal cuando el umbral se encuentra a menos de 15-20 dB HL e hipoacusia superficial cuando el umbral auditivo está entre 20-40 dB HL, lo que representa alteraciones mínimas pero significativas en la adquisición y desarrollo del lenguaje. La hipoacusia media se define como el umbral auditivo que se encuentra entre 40-70 dB HL y la palabra hablada sólo se percibe cuando es emitida a alta intensidad, lo que implica dificultades para la comprensión y desarrollo del lenguaje. La hipoacusia profunda representa un umbral que se encuentra entre los 70-90 dB HL, el paciente sólo escucha gritos y obtiene con el tiempo la habilidad de utilizar la lectura labial-facial; además es imprescindible el empleo de audífonos para working with the Neonatology Service timely interventions had been achieved to greatly reduce the effect of risk factors on patient's auditory results.

Keywords: Hearing screening, deafness, hearing loss, newborns, risk factors.

Level of evidence: III

amplificar el sonido y apoyo de terapia de lenguaje para lograr la adquisición de éste. La anacusia audiométrica o restos auditivos es la pérdida auditiva que supera los $90 \mathrm{~dB}$ HL, lo que ocasiona importantes alteraciones en el desarrollo global del niño y afecta a las funciones de alerta y orientación, estructuración y desarrollo intelectual y social.

Por topografía, la hipoacusia es clasificada como conductiva o neurosensorial. La primera representa una alteración en la transmisión del sonido a través del oído externo o el oído medio, mientras que la segunda es producto de una lesión en el oído interno o en la vía auditiva. Existe un tercer tipo denominado mixto, en el cual el compromiso de la audición está condicionado por causas sensoriales y conductivas., ${ }^{4,5}$

La sordera infantil tiene amplias implicaciones a largo plazo, las cuales incluyen desarrollo social y emocional, logros educativos y oportunidades vocacionales posteriores, incluso en los casos de compromiso leve de la audición uni o bilateral. ${ }^{6}$ Dependiendo de la edad de inicio, la discapacidad auditiva se clasifica como perlingual o postlingual, por lo que la identificación temprana es esencial y conduce a una reducción de las complicaciones que implica la sordera para un individuo. ${ }^{7}$

Se calcula que a nivel mundial $0.1 \%$ de los recién nacidos de término y con peso adecuado al nacimiento padecen algún tipo de compromiso auditivo, mientras que esta misma cifra se incrementa hasta 10 veces para los recién nacidos pretérmino y con bajo peso al nacer, que además cuentan con factores de riesgo y requieren hospitalización en las UCIN. 8,9

La OMS, en 2018, documentó que existen 466 millones de personas en el mundo que padecen pérdida de audición discapacitante ( $5 \%$ de la población), de las cuales 34 millones son niños y, de éstos, $60 \%$ de los casos de pérdida de audición tiene una etiología prevenible. Se estima que para el año 2050, más de 900 millones de personas ( 1 de cada 10) sufrirán pérdida de audición discapacitante. ${ }^{10}$ 
En cohortes internacionales de las grandes instituciones de Estados Unidos y Europa se reportan cifras de $2-3 \%$ de pacientes con sordera que cuentan con factores de riesgo al nacimiento, lo que permite hacer una adecuada y oportuna detección e intervención de oportunidades como al resto de los niños sanos. ${ }^{11,12}$

La detección temprana de sordera es esencial en la infancia; es uno de los objetivos principales en la pediatría nacional desde los años noventa, y los métodos utilizados para el tamizaje auditivo neonatal están dirigidos a detectar y prevenir la pérdida auditiva en etapa temprana de la vida.

Los casos desatendidos de pérdida de audición representan un costo mundial anual de 750,000 millones de dólares, además los gastos que representa un niño con compromiso auditivo son tres veces mayores comparados con los de un niño con audición normal; sin embargo, las intervenciones destinadas a prevenir, detectar y tratar la pérdida de la audición son económicas y pueden resultar beneficiosas para los afectados. ${ }^{13}$

Es importante el seguimiento de los pacientes con factores de riesgo de hipoacusia; la Norma Oficial Mexicana para la detección de hipoacusia en el recién nacido enlista factores identificados por la Joint Committee on Infant Hearing y verificados por la Comisión para la Detección Precoz de la Hipoacusia, entre los que se encuentran: los antecedentes familiares de hipoacusia neurosensorial, infecciones maternas durante el embarazo confirmadas o sospechadas (tales como citomegalovirus, toxoplasmosis, herpes, rubeola, sífilis o virus de inmunodeficiencia humana), así como rasgos clínicos del recién nacido que sugieran dichos padecimientos; también se incluyen las anomalías craneofaciales y de cuello que afecten la línea media o las estructuras relacionadas con el oído; recién nacidos pretérmino menores de 32 semanas de edad gestacional o con un peso menor a 1,500 gramos, hiperbilirrubinemia, que requiera exanguinotransfusión o mayor de $20 \mathrm{mg} / \mathrm{dL}$, medicamentos y otros productos ototóxicos; isótopos radiactivos, neuroinfección, accidente hipóxico-isquémico en los que se califica al recién nacido con un Apgar menor a cuatro en el primer minuto y menor a seis a los cinco minutos; así como encefalopatía de moderada a grave, síndrome de distrés respiratorio secundario a la aspiración de meconio, requerimiento de ventilación mecánica por más de cinco días, trauma de cráneo que afecte a la base y padecimientos sindrómicos como síndrome de Down y síndrome de Waardenburg. ${ }^{1,14-17}$
Se reporta que es posible detectar en $50-70 \%$ al recién nacido con sordera profunda mediante la realización del tamiz auditivo neonatal y por medio del estudio de emisiones otoacústicas (EOA) para la evaluación de la vía periférica y confirmación de ésta, junto con evaluaciones de la vía central a través de potenciales evocados auditivos del tallo cerebral (PEATC). ${ }^{13,4}$

El tamiz auditivo neonatal es el paso inicial en el protocolo de la detección oportuna de hipoacusia-sordera. Desde 1993 hasta la actualidad, se ha implementado este método de forma universal, el cual debe ser realizado antes de los tres meses de edad para efectuar un diagnóstico temprano y para que los niños con compromiso auditivo se identifiquen antes de los seis meses de edad con la finalidad de una intervención adecuada e inclusión al sistema educativo. ${ }^{4,18-22}$

El objetivo es describir el resultado del diagnóstico temprano de sordera en el recién nacido con factores de riesgo en el Centro Médico ABC.

\section{MÉTODO}

Se realizó un estudio observacional, descriptivo, retrospectivo, transversal y retrolectivo en el Servicio de Neurofisiología Clínica y Audiología perteneciente al Centro Médico ABC Campus Santa Fe, mediante la revisión de la base de datos de pacientes hospitalizados en la UCIN y UTIN, en el periodo comprendido de enero de 2010 a mayo de 2018.

Los criterios de inclusión fueron: recién nacidos en el Centro Médico ABC hospitalizados en la UCIN y UTIN, con factor de riesgo perinatal con fecha de nacimiento en el periodo comprendido entre enero de 2010 y mayo de 2018, aquéllos explorados con equipo Maico y otodinamics para EOA, que no pasaron la primera prueba de tamizaje y fueron explorados con el equipo Madsen para EOA, y/o con realización de estudios subsecuentes con equipo XLTEK o Cadwell para PEATC y con equipo Grason-Stadler modelo GSI Tympstar para timpanometría en el Servicio de Audiología y Neurofisiología, y ambos sexos.

Los criterios de exclusión fueron: recién nacidos en cunero fisiológico y aquéllos sin factores de riesgo perinatal. Además, los criterios de eliminación fueron: recién nacidos que no contaron con expediente completo.

El tamaño de la muestra fue a conveniencia e incluyó a todos los pacientes hospitalizados en la UCIN y UTIN nacidos en el Centro Médico ABC Campus Santa Fe en el periodo previamente establecido. Se realizó la recolección de datos por medio de una fuen- 
te secundaria y mediante la revisión de resultados de tamiz auditivo neonatal y de expediente clínico, recabándose los siguientes datos: edad, sexo, factores de riesgo perinatal y oído con alteración, así como estudios subsecuentes. Todo ello se registró en la hoja de recolección de datos.

Este estudio se rigió por los aspectos básicos de toda investigación biomédica, por los principios científicos y éticos que justifican la investigación, así como la información que se recolectó, fue de carácter confidencial, manteniéndose en todo momento el anonimato de los pacientes y sólo fue utilizada para fines del estudio. Se realizó el análisis estadístico de los resultados por medio de descripción con base en medidas de tendencia central utilizando el paquete estadístico SPSS v21.0.

\section{RESULTADOS}

Durante el periodo de enero de 2010 a mayo de 2018, se registraron en el Centro Médico ABC Campus Santa Fe un total de 11,698 nacidos vivos. La mayor frecuencia de nacimientos ocurrió en el año 2017 con 1,550 (10.4\%), seguida por el año 2016 con 1,473 $(9.9 \%)$ y por el año 2014 con $1,448(9.7 \%)$. Del total de recién nacidos vivos, 7,703 (51.7\%) corresponden al sexo masculino y 7,198 (48.3\%) al sexo femenino; se realizó tamiz auditivo al total de la población.

Dentro de estos pacientes, se encontró un total de 1,735 ingresos a los servicios de la UCIN y UTIN, lo que representa $14.8 \%$ de la población total, de los cuales se excluyeron 313 pacientes, entre los que se enlistan 32 nacidos fuera del hospital, 47 defunciones y 234 pacientes con expedientes no disponibles para su localización y revisión de factores de riesgo; esto implica más de $10 \%$ de los datos requeridos ausentes.

Se revisaron los expedientes electrónicos de 1,422 pacientes, de ellos 848 (59.6\%) ingresaron a la UCIN y $574(40.3 \%)$ a la UTIN, $54.8 \%$ fue correspondiente al sexo masculino y $45.2 \%$ al sexo femenino.

Los pacientes contaron con estancia intrahospitalaria variable, con un rango de un día hasta un máximo de 271 días, y promedio de estancia de 51.4 días.

La distribución de los pacientes por edades gestacionales mostró un total de 589 (41.1\%) recién nacidos considerados de término y 833 (58.6\%) prematuros, de los cuales 360 (25.3\%) se encontraban en el grupo de riesgo de compromiso auditivo según lo publicado en la literatura, al encontrarse clasificados como prematuros moderados y extremos.

En relación con el peso al nacimiento, 1,029 (72.4\%) contaron con un peso por arriba de $1,500 \mathrm{~g}$ y
$393(27.6 \%)$ prematuros contaban con un peso inferior a 1,500 g, lo que los coloca en riesgo de compromiso auditivo.

Respecto a la calificación de Apgar al nacimiento, se obtuvieron los siguientes datos: $1,092(76.8 \%)$ recién nacidos tuvieron una calificación mayor a ocho y $330(23.2 \%)$ presentaron algún grado de asfixia, de los cuales $202(14.2 \%)$ presentaron asfixia leve, 88 (6.2\%) asfixia moderada y $40(2.8 \%)$ asfixia grave.

Dentro de los factores de riesgo encontrados, en primer lugar se encuentra el uso de fármacos ototóxicos en 605 pacientes (42.5\%), seguido de hiperbilirrubinemia en $458(32.2 \%)$, taquipnea transitoria del recién nacido en 450 (31.6\%), hipertensión arterial pulmonar en 419 (29.5\%), ventilación mecánica en $385(27.1 \%)$, persistencia del conducto arterioso en 380 (26.7\%), sepsis en 320 (22.5\%), enfermedad de membrana hialina en $290(20.4 \%)$, síndrome de distrés respiratorio en $131(9.2 \%)$, hemorragia intraventricular en 117 (8.2\%), malformaciones del sistema nervioso central o síndromes con asociación a hipoacusia ya conocidos en 18 (1.3\%) y otros encontrados en la revisión que fueron tomados en cuenta por condicionar comorbilidades relacionadas en 51 pacientes $(3.6 \%)$.

Asimismo, 795 (55.9\%) neonatos requirieron el uso de fármacos ototóxicos, de los cuales a 151 $(10.6 \%)$ pacientes se administraron aminoglucósidos como primera línea de tratamiento para sepsis neonatal, furosemida a $147(10.3 \%)$ pacientes para el manejo de líquidos en patologías como persistencia del conducto arterioso, hipertensión arterial pulmonar y otras comorbilidades importantes, y por último se administró indometacina a $119(8.4 \%)$ pacientes, el cual es el antiinflamatorio no esteroideo con mayor uso para el cierre del conducto arterioso.

En la población estudiada se encontró un total de 385 pacientes $(27.1 \%)$ que requerían apoyo ventilatorio, de los cuales $260(18.3 \%)$ requirieron entre cinco y 10 días de ventilación, 88 (6.2\%) más de 10 días y $37(2.6 \%)$ menos de cinco días.

Otro factor de riesgo auditivo que se ha estudiado es la lesión cerebral; en nuestra población tuvimos un total de 116 neonatos (8.2\%) con diagnóstico de hemorragia intraventricular, 53 (3.7\%) grado 1, 42 (2.9\%) grado 2,16 (1.1\%) grado 3 , y finalmente cinco $(0.3 \%)$ grado 4 , todos clasificados como prematuros.

Las malformaciones congénitas, sobre todo de la línea media, y algunos síndromes descritos tienen una importante relación con el compromiso de la audición, entre los que se encontraron: principalmente, síndrome de Down con 10 (0.7\%) pacientes, seguido 
de labio y paladar hendido en tres $(0.2 \%)$ recién nacidos, así como síndrome de DiGeorge, síndrome de Crouzon, microcefalia, hidrocefalia y mielomeningocele con presencia de un solo paciente $(0.07 \%)$, respectivamente.

Otras patologías encontradas en la revisión de casos que no precisamente están relacionadas con compromiso auditivo, pero que sus complicaciones y tratamientos llevan a los pacientes a contar con factores de riesgo para la adquisición de éste, son: las patologías cardiopulmonares (neumotórax, neumomediastino, anomalía de Ebstein, tetralogía de Fallot, ventrículo único, atresia pulmonar, agenesia de válvulas pulmonares, extrasístoles, transposición de grandes vasos) en 38 pacientes (2.7\%), patología digestiva (hernia diafragmática, atresia esofágica, atresia duodenal) en cinco $(0.3 \%)$, trauma obstétrico en cuatro $(0.2 \%)$, epilepsia en dos $(0.1 \%)$, laringomalacia en uno $(0.07 \%)$ y fibrosis quística en uno $(0.07 \%)$.

Con base en los datos previos, contamos con una idea clara del panorama de factores de riesgo que poseen los recién nacidos en este centro médico; dichos diagnósticos implican múltiples manejos e intervenciones que agregan más comorbilidades, las cuales representan factores de riesgo ampliamente estudiados para los pacientes de este grupo de edad.

El tamiz auditivo neonatal realizado de forma inicial por medio de EOA encontró que del total de pacientes estudiados de UCIN-UTIN, 35 (2.5\%) de los recién nacidos no pasaron la primera prueba, representados por $18(1.3 \%)$ pacientes del sexo femenino y 17 (1.2\%) del sexo masculino, encontrándose 23 pacientes (1.6\%) con uno a dos factores de riesgo y 12 $(0.8 \%)$ pacientes con más de dos factores de riesgo, los cuales correspondieron a factores ototóxicos en 16 (1.1\%), hiperbilirrubinemia en 14 (1\%), ventilación mecánica en $13(0.9 \%)$, enfermedad de membrana hialina en $11(0.8 \%)$, taquipnea transitoria del recién nacido en $10(0.7 \%)$, hipertensión arterial pulmonar en nueve $(0.6 \%)$, sepsis en seis $(0.4 \%)$, hemorragia intraventricular en cinco $(0.3 \%)$, peso bajo al nacer < $1,500 \mathrm{~g}$ en cinco $(0.3 \%)$ y asfixia grave, asfixia moderada, labio y paladar hendido con frecuencia de uno solo $(0.07 \%)$ por cada uno.

De los pacientes que no pasaron el tamiz auditivo inicial, $22(1.5 \%)$ no pasaron la prueba en ambos oídos, siete $(0.5 \%)$ en el oído derecho y seis $(0.4 \%)$ en el oído izquierdo.

Al total de los pacientes que no pasaron el primer examen de tamiz auditivo, se les realizó un estudio consecutivo por medio de EOA en el Servicio de Audiología a los 15 días de vida extrauterina, en donde
15 (1\%) pacientes pasaron la prueba en ambos oídos, ocho $(0.6 \%)$ no pasaron y $12(0.8 \%)$ no se presentaron a la realización de esta prueba. De los recién nacidos que no pasaron el segundo tamizaje, cuatro $(0.3 \%)$ no pasaron en ambos oídos, tres $(0.2 \%)$ en oído derecho y uno $(0.07 \%)$ en oído izquierdo, representado por cinco $(0.3 \%)$ pacientes del sexo masculino y tres $(0.2 \%)$ del sexo femenino. Se encontraron uno a dos factores de riesgo en seis pacientes $(0.4 \%)$ y más de dos factores de riesgo en dos $(0.1 \%)$ pacientes; en orden de frecuencia, tres pacientes presentaron hipertensión arterial pulmonar $(0.2 \%)$, taquipnea transitoria del recién nacido en tres $(0.2 \%)$, uso de ototóxicos en tres $(0.2 \%)$, hiperbilirrubinemia en dos $(0.1 \%)$, labio y paladar hendido, enfermedad de la membrana hialina, ventilación mecánica, peso bajo al nacer $<1,500$ g y sepsis con frecuencia de uno $(0.07 \%)$ cada uno.

Siguiendo con el protocolo que indica el algoritmo de evaluación de sordera en el recién nacido, se realizaron estudios de control para seguimiento correspondiente, sólo dos $(0.1 \%)$ de ocho recién nacidos con alteración acudieron a seguimiento. Se realizó estudio de PEATC, con presencia de un $(0.07 \%)$ paciente con respuesta anormal indicativa de hipoacusia profunda (90 dB) en ambos oídos. Como factor de riesgo este paciente presentó taquipnea transitoria del recién nacido y un $(0.07 \%)$ paciente con respuesta normal en ambos oídos tuvo labio y paladar hendido. En ambos casos el estudio de la timpanometría fue normal.

Se encontraron 1,403 $(98.6 \%)$ recién nacidos hospitalizados en la UCIN-UTIN con audición normal, uno $(0.07 \%)$ con alteración en la audición y 18 (1.3\%) sin registro de realización de pruebas consecutivas diagnósticas.

De los recién nacidos con audición normal, 772 (55\%) fueron del sexo masculino y $631(45 \%)$ del sexo femenino. Sólo un $(0.07 \%)$ paciente del sexo femenino, que tuvo hipoacusia profunda en ambos oídos, presentó taquipnea transitoria del recién nacido como único factor de riesgo.

\section{DISCUSIÓN}

En el presente estudio se describen los resultados correspondientes a la realización de tamiz auditivo neonatal de todos los pacientes del Centro Médico ABC ingresados a los servicios de la UCIN y UTIN en el periodo de enero de 2010 a mayo de 2018, secundarios a múltiples causas previamente descritas.

En comparación con los resultados de Carranza y colaboradores ${ }^{23}$ en su estudio de seguimiento de recién nacidos sin factores de riesgo para hipoacusia en 
2013, en el que se reporta una prevalencia de $0.1 \%$ de compromiso auditivo, en nuestro estudio encontramos $0.07 \%$, lo que corresponde al mismo rango que los pacientes sin factores de riesgo.

En relación con la edad gestacional y el peso al nacimiento, van Dommelen y su equipo, ${ }^{23}$ en 2015 , encontraron que la prevalencia de compromiso auditivo se incrementaba con la disminución de las semanas de edad gestacional de 1.2 a $7.5 \%$ entre las 24-31 semanas, así como con la disminución del peso al nacimiento de 1.4 a $4.8 \%$, esto es, de 750 a 1,500 g; en nuestra población, aun con dichas características, no se reprodujeron estos resultados.

Respecto a los pacientes sometidos a episodios de hipoxia, independientemente de la causa, la literatura reporta resultados contrastantes, muchos de éstos sin mayores especificaciones de la forma de evaluación del paciente ni la definición conceptual de hipoxia en cada centro. Muchos de estos resultados no se han logrado reproducir. En 2007 Hille y su grupo, ${ }^{24}$ en el Reino Unido, encontraron que la asfixia severa en menores de 30 semanas de gestación (SDG) y con un peso menor a 1,000 g tenía una gran relación con la pérdida de la audición; nuestra población incluyó pacientes con características heterogéneas, sin embargo, en nuestro estudio sólo dos pacientes contaban con dichas características y no se encontró en ellos compromiso auditivo.

Gran parte de nuestros pacientes fueron sometidos a apoyo ventilatorio. Se clasificó, según Vohr y colabadores $^{25}$ en su revisión del año 2000 , como factor de riesgo para sordera más de cinco días de ventilación mecánica, lo mismo estipulado en la NOM para de detección de hipoacusia en recién nacidos. ${ }^{1}$ En la revisión de la literatura encontramos que 9.6 días o más de ventilación mecánica son considerados de riesgo para hipoacusia en pacientes con factores de riesgo comparados con los controles, esto incluso fue corroborado por un estudio mexicano. ${ }^{26-28} \mathrm{En}$ nuestra población dentro de los pacientes con problema auditivo, 12 contaron con este factor de riesgo, cinco con ventilación mecánica entre cinco y 10 días, seis con ventilación mecánica por más de 10 días y uno menor a cinco días; sólo cinco pacientes tuvieron seguimiento sin presencia de alteración auditiva.

Un total de 116 pacientes fueron diagnosticados con algún grado de hemorragia intraventricular. Meyer y su equipo, ${ }^{29}$ en 1999, encontraron que los grados de hemorragia intraventricular más graves no fueron un factor de riesgo para hipoacusia en un estudio con edades gestacionales variables y otros factores de riesgo asociados. En nuestra población, cinco pacientes tuvieron diagnóstico de hemorragia intraventricular, de los cuales dos presentaban grado 1 , uno grado 2 y dos con grado 3 , y de ellos sólo uno aprobó el tamiz subsecuente y cuatro no acudieron al seguimiento.

En los pacientes con diagnóstico de hiperbilirrubinemia, los resultados han sido controversiales, mientras que los pacientes con cifras mayores al límite conocido como factor de riesgo resultaron sin alteraciones auditivas, los pacientes con cifras por debajo del riesgo resultaron con alteraciones en este sentido, aún sin resultados concluyentes en la literatura. Rhee y colegas, ${ }^{30}$ en 1999, estudiaron la asociación de hiperbilirrubinemia con hipoacusia en pacientes con menos de 31 semanas de gestación y con cifras de hiperbilirrubinemia $>26 \mathrm{mg} / \mathrm{dL}$, sin considerar los factores de riesgo coexistentes, sólo dos pacientes resultaron con compromiso auditivo; esto está relacionado con el tiempo de espera para la realización de exanguinotransfusión. En nuestra población, la hiperbilirrubinemia se encontró en $32.2 \%$ de los recién nacidos de la UCIN-UTIN, pero no se cuenta con las cifras de bilirrubinas al momento de realizar el diagnóstico de la gran mayoría de ellos, sólo con los controles posteriores al inicio de la fototerapia. No está documentada la realización de ningún procedimiento de exanguinotransfusión. Es de suma importancia protocolizar el uso de fototerapia en los pacientes, ya que aún en países de primer mundo se continúa teniendo disparidad en la utilización de este recurso.

Las infecciones perinatales están altamente relacionadas con la pérdida auditiva, y son dependientes de la etapa en la que los pacientes son infectados, y así las secuelas se vuelven un tanto más graves. En la etapa perinatal de infecciones adquiridas en relación con el canal vaginal o con relación de las vías urinarias de las madres, el recién nacido puede ser infectado in utero por citomegalovirus y rubéola, entre otros; mientras que posterior al nacimiento pueden sufrir complicaciones infecciosas secundarias a sus estancias hospitalarias. Dentro de nuestra población se encontraron 320 pacientes con sospecha de sepsis, sin detalles claros de la etiología. Entre los pacientes con problema auditivo, sólo seis contaban con este factor de riesgo, de los cuales tres se perdieron al seguimiento, mientras que los otros tres contaron con pruebas de tamiz y de seguimiento normales.

Una gran variedad de fármacos está asociada con ototoxicidad, entre éstos están: los antibióticos como aminoglucósidos, los diuréticos de asa como furosemida, los antiinflamatorios no esteroideos como indometacina, entre otros. Los mecanismos por los cuales 
éstos condicionan daño audiológico continúan siendo investigados, ya que hasta el momento los estudios son controversiales tanto en poblaciones de término como pretérmino, así como la influencia de multidrogas ototóxicas en el resultado final de la audición en los pacientes. En nuestro registro encontramos un total de 795 recién nacidos expuestos a uno o varios ototóxicos, sin contar el número de días de exposición ni la dosis total acumulada. Entre los pacientes con falla en el tamiz auditivo neonatal, se encontró que la mayoría contaba con aplicación de ototóxicos durante su estancia intrahospitalaria; sin embargo, todos ellos pasaron las pruebas ya sea de forma inicial o subsecuente.

Además de los factores de riesgo ya conocidos, en nuestra población encontramos algunos pacientes con padecimientos sindromáticos y malformaciones relacionados con trastornos auditivos. En los pacientes con falla en nuestro tamizaje sólo se encontraba un paciente con labio y paladar hendido, el cual pasó el tamiz auditivo en el seguimiento y un paciente con síndrome de Down se perdió en el seguimiento. El resto de los pacientes aprobó el tamiz auditivo.

Vohr y colaboradores, ${ }^{25}$ en el año 2000 , reportaron la relación del número de factores de riesgo con el resultado final de hipoacusia sin conclusiones claras, estableciendo que sus pacientes con alteración auditiva contaban por lo menos con un factor de riesgo para ésta en 59\%, mientras que $26 \%$ contaba con más de dos factores de riesgo relacionados. En nuestra población con tamiz auditivo alterado, seis recién nacidos contaban con uno a dos factores de riesgo y dos recién nacidos tenían dos o más factores de riesgo para compromiso auditivo. La paciente con alteración final en los PEATC contaba con sólo un factor de riesgo.

La intervención del Servicio de Neonatología se realizó de forma oportuna, por lo que los factores de riesgo no afectaron significativamente el resultado final del tamiz auditivo de la población neonatal del Centro Médico ABC, aunada a la atención eficaz de los embarazos de las pacientes, la atención certera de sus complicaciones en el embarazo y la calidad de vida con la que cuentan.

\section{CONCLUSIÓN}

Se diagnosticó con hipoacusia profunda al recién nacido con factores de riesgo en el Centro Médico ABC. La frecuencia de audición normal fue de 1,403 recién nacidos en la UCIN-UTIN, con predominio del sexo masculino. En el tamiz auditivo por medio de EOA con resultados alterados, predominó el sexo masculino, con mayor frecuencia de alteración en ambos oídos, seguida de la alteración en el oído derecho.

Los factores de riesgo que predominaron fueron: la administración de ototóxicos, hiperbilirrubinemia y ventilación mecánica. En los PEATC, se presentó alteración de tipo hipoacusia profunda en ambos oídos. En un paciente del sexo femenino hubo taquipnea transitoria del recién nacido como factor de riesgo. A lo largo de diferentes etapas del diagnóstico, en 1.3\% del total de los recién nacidos no se les realizó pruebas consecutivas de detección, ya que no acudieron a éstas, ignorándose el motivo. Estos resultados son similares a los reportados por nuestro grupo en recién nacidos sin factores de riesgo conocidos para hipoacusia.

Es de suma importancia continuar con el seguimiento de los pacientes en quienes el tamiz auditivo neonatal se encuentra alterado hasta asegurarnos de la presencia de resultados favorables o de la canalización de éstos hacia las intervenciones correspondientes, así como contar con expedientes clínicos con datos detallados de los factores de riesgo y seguimiento de los pacientes ingresados en los servicios de neonatología, incluyendo la UCIN-UTIN.

\section{BIBLIOGRAFÍA}

1. Secretaría de Salud. Guía de Práctica Clínica GPC. Detección de Hipoacusia en el recién nacido en el primer nivel de atención. [Consultado 30/01/2018]. Disponible en: http: //www. cenetec.salud.gob.mx/descargas/gpc/CatalogoMaestro/055_ GPC_Hipoacusiaped/SSA_055_08_GRR.pdf.

2. Fontané-Ventura J. Déficit auditivo. Retraso en el habla de origen audígeno. Rev Neurol. 2005; 41 (1): S25-S37.

3. Chávez-Delgado ME, Álvarez-Raygoza Y, Celis de la-Rosa A, Virgen-Enciso M, Castro-Castañeda S. Déficit auditivo en pacientes atendidos en otorrinolaringología del IMSS en Guadalajara. Rev Med Inst Mex Seguro Soc. 2008; 46 (3): 315-322.

4. American Academy of Pediatrics (1995) Joint Committee on Infant Hearing 1994 Position Statement. Pediatrics. 1995; 95 (1): 152-156.

5. Comisión para la Detección Precoz de la Hipoacusia. Detección Precoz de la Sordera. Dossier Informativo. [Consultado 30/01/2018]. Disponible en: http: //www.msal.gob.ar/images/ stories/bes/graficos/0000000232cnt-detecprecoz.pdf

6. Tharpe AM. Unilateral and mild bilateral hearing loss in children: past and current perspectives. Trends Amplif. 2008; 12 (1): 7-15.

7. Hilgert N, Smith RJ, Van Camp G. Forty-six genes causing nonsyndromic hearing impairment: which ones should be analyzed in DNA diagnostics? Mutat Res. 2009; 681 (2): 189-196.

8. Wood NS, Marlow N, Costeloe K, Gibson AT, Wilkinson AR. Neurologic and developmental disability after extremely preterm birth. N Engl J Med. 2000; 343 (6): 378-384.

9. Roberts G, Anderson PJ, Doyle LW, Victorian Infant Collaborative Study Group. Neurosensory disabilities at school age in geographic cohorts of extremely low birth weight children born between the 1970s and the 1990s. J Pediatr. 2009; 154 (6): 829-834. 
10. Organización Mundial de la Salud [Internet]. Sitio web mundial en español. [Actualizado 15/03/2018; citado 30/03/2018]. Sordera y pérdida de la audición; [aprox. 6 pp.]. Disponible en: http: //www.who.int/es/news-room/fact-sheets/ detail/deafness-and-hearing-loss.

11. National Institutes of Health Consensus Development Conference Statement. Early identification of hearing impairment in infants and young children. Int J Pediatr Otorhinolaryngol. 1993; 11 (1): 1-24.

12. Yoon PJ, Price M, Gallagher K, Fleisher BE, Messner AH. The need for long-term audiologic follow-up of neonatal intensive care unit (NICU) graduates. Int J Pediatr Otorhinolaryngol. 2003; 67 (4): 353-357.

13. Schroeder L, Petrou S, Kennedy C, McCann D, Law C et al. The economic costs of congenital bilateral permanent childhood hearing impairment. Pediatrics. 2006; 117 (4): 1101-1112.

14. Gaceta CENETEC. Implantes cocleares. 2a Época, Año 1, No 1, abril de 2009-Julio de 2009. [Consultado 05/02/2018]. Disponible en: http: //www.cenetec.gob.mx/interior/ gaceta2_1_1/sumario.html

15. Secretaría de Salud. Guía de Práctica Clínica. Hipoacusia neurosensorial bilateral e implante coclear. [Consultado 01/02/2018]. Disponible en: http: //www.cenetec.salud.gob.mx/ descargas/gpc/CatalogoMaestro/396_IMSS_10_hipoacusia_ neurosensorial/EyR_IMSS_396_10.pdf

16. Martínez CG, Valdez GM. Detección oportuna de la hipoacusia en el niño. Acta Pediatr Mex. 2003; 24 (3): 176-180.

17. Dunmade AO, Dunmade AD, Taiwo OA, Tomori AR, Komolafe TM. A software system for diagnosis and classification of deafness. European J Scientific Research. 2009; 25 (4): 597-605.

18. Programa de Acción Específico 2007-2012. Tamiz auditivo neonatal e intervención temprana. México, D.F. [Consultado 12/03/2018]. Disponible en: http://www.anmm.org.mx/ publicaciones/CAnivANM150/L13-Tamiz-auditivo neonatal.pdf

19. National Institutes of Health. Early identification of hearing impairment in infants and young children. [Consultado 31/01/2018]. Disponible en: https: //consensus.nih.gov/1993/1 993hearinginfantschildren092html.htm

20. Toral-Martiñón R, Collado-Corona MA, ShkurovichZavslasky M. Diagnóstico temprano de sordera por emisiones otoacústicas en el recién nacido. An Med Asoc Med Hosp ABC. 1997; 42 (3): 111-113.
21. Norma Oficial Mexicana NOM-034-SSA2-2013, Para la prevención y control de los defectos al nacimiento. [Consultado 10/03/2018]. Disponible en: http: //dof.gob.mx/nota_detalle.ph p? codigo $=5349816 \&$ fecha $=24 / 06 / 2014$

22. Carranza-Alva, Toral-Martiñón R, Shkurovich-Bialik P, Schabes-Rostenberg M. Resultados del diagnóstico temprano de sordera en recién nacidos sin factores de riesgo. An Med (Mex). 2016; 61 (2): 93-97.

23. van Dommelen P, Verkerk PH, van Straaten HL; Dutch Neonatal Intensive Care Unit Neonatal Hearing Screening Working Group. Hearing loss by week of gestation and birth weight in very preterm neonates. J Pediatr. 2015; 166 (4): 840843.e1.

24. Hille ET, van Straaten HI, Verkerk PH, Dutch Neonatal Intensive Care Unit Neonatal Hearing Screening Working Group. Prevalence and independent risk factors for hearing loss in NICU infants. Acta Paediatr. 2007; 96 (8): 11551158.

25. Vohr BR, Widen JE, Cone-Wesson B, Sininger YS, Gorga MP et al. Identification of neonatal hearing impairment: characteristics of infants in the neonatal intensive care unit and well-baby nursery. Ear Hear. 2000; 21 (5): 373-382.

26. Bergman I, Hirsch RP, Fria TJ, Shapiro SM, Holzman I et al. Cause of hearing loss in the high-risk premature infant. J Pediatr. 1985; 106 (1): 95-101.

27. Martínez-Cruz CF, Poblano A, Fernández-Carrocera LA. Risk factors associated with sensorineural hearing loss in infants at the neonatal intensive care unit: 15-year experience at the National Institute of Perinatology (Mexico City). Arch Med Res. 2008; 39 (7): 686-694.

28. De Capua B, De Felice C, Constantini D, Bagnoli F, Passali D. Newborn hearing screening by transient evoked otoacoustic emissions: analysis of response as a function of risk factors. Acta Otorhinolaryngol Ital. 2003; 23 (1): 16-20.

29. Meyer C, Witte J, Hennecke KH, Schunck KU, Maul K et al. Neonatal screening for hearing disorders in infants at risk: incidence, risk factors, and follow-up. Pediatrics. 1999; 104 (4): 900-904.

30. Rhee CK, Park HM, Jang YJ. Audiologic evaluation of neonates with severe hyperbilirubinemia using transiently evoked otoacoustic emissions and auditory brainstem responses. Laryngoscope. 1999; 109 (12): 2005-2008. 